\title{
Olfactory neuroblastoma: A case report
}

\author{
GONCA HANEDAN USLU ${ }^{1}$, EMINE CANYILMAZ ${ }^{2}$, AHMET YASAR ZENGIN ${ }^{1}$, SEVDEGUL MUNGAN $^{3}$, \\ ADNAN YONEY ${ }^{2}$, OSMAN BAHADIR ${ }^{4}$ and HUSEYIN GOCMEZ ${ }^{1}$ \\ ${ }^{1}$ Department of Radiation Oncology, Faculty of Medicine, Kanuni Research and Education Hospital, Trabzon 61040; \\ Departments of ${ }^{2}$ Radiation Oncology, ${ }^{3}$ Pathology and ${ }^{4}$ Otorhinolaryngology, Faculty of Medicine, \\ Karadeniz Technical University, Trabzon 61080, Turkey
}

Received November 17, 2014; Accepted October 5, 2015

DOI: $10.3892 / 01.2015 .3821$

\begin{abstract}
Olfactory neuroblastoma $(\mathrm{ON})$ is a rare type of malignant neoplasm originating from the olfactory neuroepithelial cells of the nasal cavity. $\mathrm{ON}$ is also known as esthesioneuroblastoma or neuroendocrine carcinoma. The malignancy accounts for $<3 \%$ of tumors originating in the nasal cavity. Through the nasal cavity, ON may infiltrate the sinuses, the orbit and the cranium. The tumor is characterized by a pattern of slow growth and local recurrences. Treatment options are surgical excision or surgery combined with a radiotherapy (RT) and/or chemotherapy combination treatment. The present study reports the case of a 69-year-old patient with a mass in the nasal cavity who was treated by combined surgical excision and RT. The literature for ON and the treatment of the tumor are also discussed.
\end{abstract}

\section{Introduction}

Olfactory neuroblastoma (ON) is an uncommon malignant nasal tumor which is originated from neuroectoderm. It comprises $\sim 2 \%$ of all sinonasal tract tumors. Its incidence is $\sim 0.4$ per million in population $(1,2)$. The most common symptoms of ON are unilateral nasal obstruction (70\%), and epistaxis (50\%). Other symptoms include headaches, pain, excessive lacrimation, rhinorrhea, anosmia and changes in vision. ON originates from olfactory epithelium; however, ON rarely causes anosmia $(5 \%)(3,4)$. ON may histologically mimic a number of types of tumor within the sinonasal tract, making it more difficult to diagnosis. The management of ON requires bicranial-facial surgical approach, trephination procedure, which is technically challenging and achieving good results are difficult. Treatment modalities for $\mathrm{ON}$

Correspondence to: Dr Emine Canyilmaz, Department of Radiation Oncology, Faculty of Medicine, Karadeniz Technical University, 2 Farabi Street, Trabzon 61080, Turkey

E-mail:dremocan@yahoo.com

Key words: esthesioneuroblastoma, olfactory neuroblastoma, surgical resection, radiotherapy are en bloc resection, extra cranial resection or surgery combined with radiotherapy (RT) and/or chemotherapy. The present study reports the case of a patient with a mass in the nasal cavity who was treated by combined surgical excision and RT.

\section{Case report}

A 69-year-old male patient presented to the Kanuni Research and Education Hospital (Trabzon, Turkey) with a mass in the nose in October 2012. Endoscopy showed the presence of a mass within the nasal cavities, causing destruction of the nasal dorsum. Upon cranial magnetic resonance imaging (MRI) a homogenous contrast-enhanced mass, $24 \times 33 \mathrm{~mm}$ in size, was observed (Fig. 1). The mass was isointense on T1-weighted imaging, and hyperintense on T2-weighted imaging and fluid attenuated inversion recovery. The mass infiltrated the nasal septum in the anterior region. A second mass was also observed posterior to the first mass. This second mass was $2 \mathrm{~cm}$ in diameter and was an intensely contrast-enhanced well-circumscribed lesion. At the superior border, the mass reached the frontal bone causing bony destruction. A biopsy from the lesion reported a diagnosis of ON (Fig. 2A and B). The patient was treated with a wide tumor excision by nasal endoscopic surgery. The post-operative pathology both lesions showed a grade II ON. Surgical margins were positive on medial canthus and negative in the base of the cranium. Immunohistochemistry results were reported as positive for neuron-specific enolase (NSE), weakly positive for chromogranin, and negative for vimentin and S-100 protein.

Follwing surgery, the patient was treated with the TomoTherapy Hi-Art System ${ }^{\circledR}$ using an intensity-modulated radiotherapy (IMRT) technique, and 66 Gy external RT with $220 \mathrm{cGy} /$ fraction was applied to the pre-operative tumor bed. The patient was followed up without using chemotherapy.

After 2 years of follow-up, a mass was palpable on the left side of the neck upon physical examination. Computed tomography revealed a hyperdense metastatic lymphadenopathy, 29x25 mm in size, in the left submandibular region (Fig. 3). Lymph nodes of $<1 \mathrm{~cm}$ in diameter were also present in the 5 cervical neck region and they were non-malignant. The patient was treated with a radical neck dissection. The pathology report recorded 16 reactive lymph nodes, and 1 lymph node that was $4 \mathrm{~cm}$ in diameter was diagnosed as an 


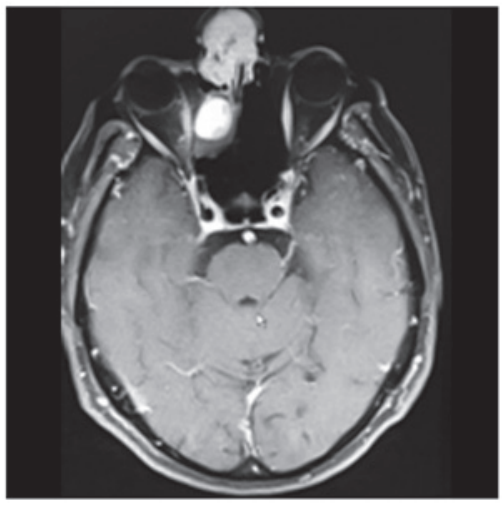

Figure 1. Cranial magnetic resonance imaging showing a homogenous contrast-enhanced mass, $24 \times 33 \mathrm{~mm}$ in size.
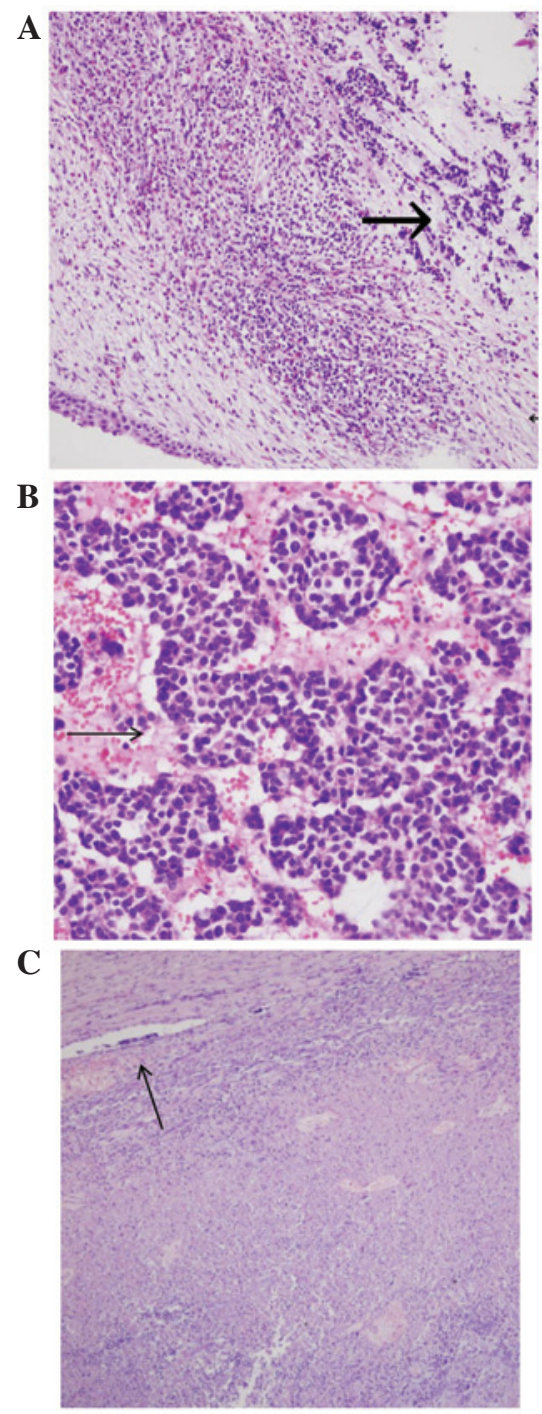

Figure 2. Hematoxylin and eosin staining. (A) Nasal mucosal epithelium (thin arrow) and neoplastic cell groups under epithelium (thick arrow). (B) Narrow cytoplasmic atypical cells with hyperchromatic nuclei (arrow). (C) Extensive necrosis in metastatic lymph node and tumor cells in neighboring lymphatics (arrow).

ON metastasis in the sub-capsular region (Fig. 2C). The immunohistochemistry results were reported as positive for NSE and chromogranin (Fig. 4), and negative for synaptophysin.

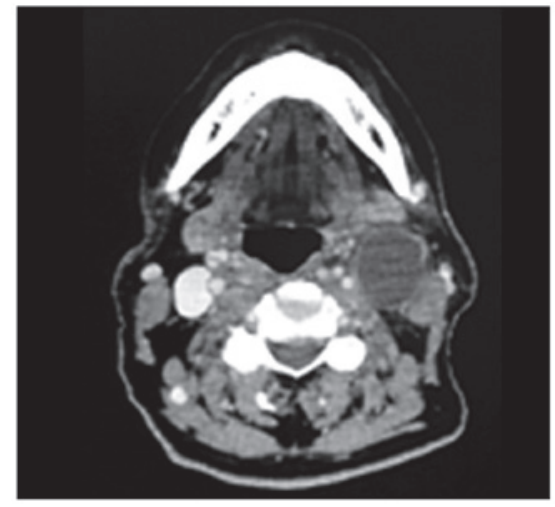

Figure 3. Neck computed tomography showing a hyperdense metastatic lymphadenopathy, 29x25 mm in size, in the left submandibular region.

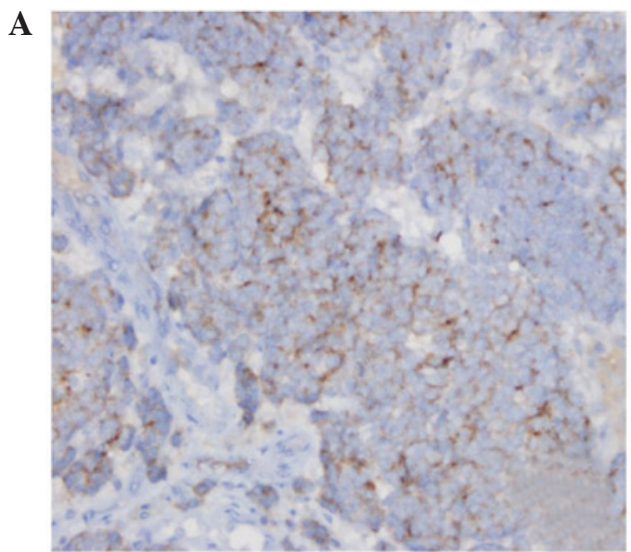

B

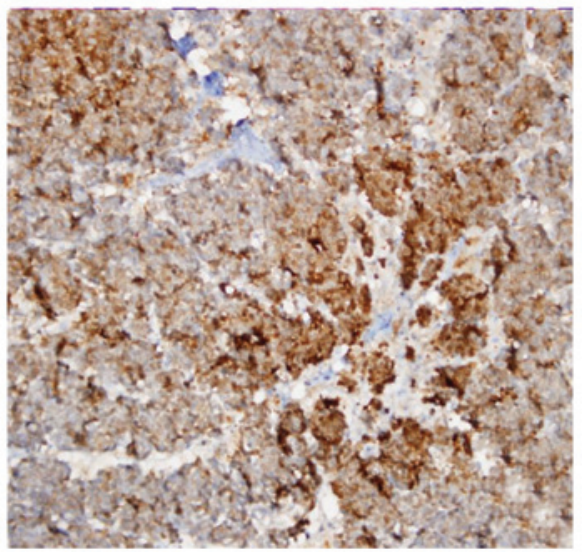

Figure 4. Immunohistochemistry. (A) Chromogranin and (B) neuron-specific enolase expression of neoplastic cells.

No distant organ metastasis or recurrence in the primary tumor region was detected. The patient was treated with the Hi-Art Tomotherapy IMRT technique after taking consideration of the prior treatment doses for regions and organs at risk. A total of $54 \mathrm{~Gy}$ external RT, with $200 \mathrm{cGy} /$ fraction, was administered to the right neck region (levels I-V), while a total of $60 \mathrm{~Gy}$ external RT, with $200 \mathrm{cGy} /$ fraction, was administered to the left neck region (level I-V). Subsequently, $100 \mathrm{mg}$ etoposide was administered for 7 consecutive, every 21 days, for 6 months. The patient is in the 3rd year after diagnosis and a complete response has been observed after post-operative treatment. 


\section{Discussion}

$\mathrm{ON}$ is a rare locally aggressive tumor (5). Although it can be found in all age groups, it occurs more commonly in the 3rd and 6th decades of life, and is present equally in each gender. The most common symptoms are one-sided nose obstruction and epistaxis, while rhinorrhea and anosmia may also occasionally accompany these symptoms. Extensive lesions may cause frontal headaches and diplopia. As earlier symptoms are not specific, $70 \%$ of patients consult their doctors with advanced-stage disease $(6,7)$. ON can spread quickly and easily into the intracranial structures via the cribriform plate. Cribriform plate and orbit involvement in ON are important prognostic factors (8). It has been reported that the cervical lymph node involvement rate is nearly $20 \%$, that the local recurrence rate is $20-30 \%$ and that the 6-month distant metastasis rate is $50 \%(9,10)$. In the present case, distant metastasis was observed 2 years after the completion of treatment. Studies have been conducted in order to evaluate treatment and prognosis of ON staging. Computed tomography and MRI are important in staging. Kadish et al (11) performed the first staging of ON using clinical evaluation and neuroradiological findings. According to this staging, stage $\mathrm{A}$ is a tumor limited to the nasal cavity, stage B is a tumor is limited to the nasal cavity and one or more of the paranasal sinuses, and stage $\mathrm{C}$ is a tumor extending from the nasal cavity and paranasal sinuses. Orbit, skull base and intracranial cavity involvement or cervical lymph node and distant metastasis is present. When patients are diagnosed, the majority are at stages $\mathrm{C}(56 \%)$ and B (40\%), while only a small portion of patients are at stage A (4\%) (12). The present case was diagnosed as stage C following radiological assessment.

Subsequent to the staging of ON, multidisciplinary approaches have been used for treatment, and no standard treatment approach has yet been established. The aim of the treatment should prevent local and regional recurrences, and distant metastasis. Treatment options consist of surgery or RT only, surgery and RT, surgery and chemotherapy combined with RT, or only chemotherapy $(13,14)$. A craniofacial resection has been suggested for all patients with frontal cranial base involvement $(4,14)$. It has been reported that, in selected patients, endoscopic sinus surgery and stereotactic radiosurgery lead to good results (15). Walch et al (10) obtained tumor control without any patient mortality by combining stereotactic radiosurgery and endoscopic sinus surgery in individuals with stage $\mathrm{B}$ and $\mathrm{C}$ disease according to the Kadish classification (11).

In the present patient, endoscopic sinus surgery with a wide local excision was performed post-diagnosis, and RT was subsequently applied. ON may metastasize and reoccur following its removal $(12,15)$, so post-operative therapy should be added to the treatment. In a study of 29 patients treated with a craniofacial resection, Aboziada and Eisbruch (16) suggested that the addition of RT to a craniofacial resection leads to recurrence in 2/13 patients, while no additional RT leads to recurrence in 11/16 patients. Even though chemotherapy and RT treatments are routine for stage C disease, the study by Benfari et al (17) indicated that RT should be applied to all patients, with the exception of cases with tumors limited to the cribriform plate without bony destruction. The most important deductions from the aforementioned studies are summarized as follows: i) RT alone is effective in $36.3 \%$ of patients. ii) Survival rates have a tendency to decrease as tumor stage increases (stage A, 100\%; stage B, 58.3\%; and stage $\mathrm{C}, 18.9 \%$ ). iii) There is no correlation between survival and radiation dose. The majority of patients who succumbed to the disease received RT doses of 50-65 Gy, as recommended in the literature. iv) The presence of palpable neck nodes and/or distant metastasis, at presentation, is a significant prognostic factor for survival. Regional lymph node metastasis was present in 6 patients at presentation, and of these, 4 patients succumbed to the disease, with a median survival time of 5.2 months; an identical outcome was noted in $2 / 2$ patients with distant metastasis. v) A variable and often prolonged natural history is characteristic of ON. This prerogative is highlighted by the 1 patient who remained alive with the disease at 120 months (17).

In another study, the 5-year local relapse-free survival rate was significantly higher for those patients who received post-operative RT (100\%) compared with surgery alone (29\%). The 5-year disease-free survival rate was $87.5 \%$ in the RT group and $31 \%$ in the group that underwent surgery alone. Regional failure was observed in 7 patients (27\%); 6 with stage B and 1 with stage $\mathrm{C}$ disease according to the Kadish classification. Nodal failure most commonly occurred at level II of the neck, with 3 patients experiencing nodal failure in the contralateral neck. Only 3 of the cases with regional failure were salvaged successfully. Due to the high rate of regional failure following a lack of elective treatment on the neck, elective nodal RT is justified in patients with Kadish stage B and C disease. These results confirmed the beneficial effect of adjuvant $\mathrm{RT}$ to the tumor bed on local control (18). However, in a retrospective analysis, Montava et al (19) emphasized that the gold-standard treatment for $\mathrm{ON}$ is craniofacial resection and that mortality is associated with RT.

In conclusion, a standard treatment for $\mathrm{ON}$ is not yet clear as the number of ON cases is limited. However, due to the $20 \%$ risk of neck metastasis in stage B and C, treatment should include a wide surgical excision and prophylactic neck irradiation should be added to the RT region. Prospective studies with a large number of patients are required in order to establish a gold-standard treatment.

\section{References}

1. Berger L,Luc R and Richard D: Olfactory esthesioneuroblastoma. Bull Assoc Fr Etude Cancer 13: 410-21,1924 (In French).

2. Bhattacharyya N, Thornton AF, Joseph MP, Goodman ML and Amrein PC: Successful treatment of esthesioneuroblastoma and neuroendocrine carcinoma with combined chemotherapy and proton radiation. Results in 9 cases. Arch Otolaryngol Head Neck Surg 123: 34-40,1997.

3. Rakes SM, Yeatts RP and Campbell RJ: Ophthalmic manifestations of esthesioneuroblastoma. Ophthalmology 92: 1749-53,1985.

4. Kutluhan A, Yilmaz N, Yakut F, Yurttaş V and Uğraş S: Treatment of olfactory neuroblastoma via subfrontal and midfacial degloving approaches: A case report. Kulak Burun Bogaz Ihtis Derg 18: 56-8, 2008 (In Turkish).

5. Fitzek MM, Thornton AF, Varvares M, Ancukiewicz M, Mcintyre J, Adams J, Rosenthal S, Joseph M and Amrein P: Neuroendocrine tumors of the sinonasal tract. Results of a prospective study incorporating chemotherapy, surgery, and combined proton-photon radiotherapy. Cancer 94: 2623-34, 2002. 
6. Kleihues P and Cavenee WK (eds): WHO Classification of Tumours of Pathology and Genetics Tumours of the Nervous System. IARC Press, Lyon, France, pp150-152, 2000.

7. Weiss SW, Goldblum JR and Enzinger: Primitive neuroectodermal tumors and related lesions. In: Enzinger and Weiss's Soft Tissue Tumors. 4th edition. Mosby Elsevier, Philadelphia, PA, pp1308-1311, 2001.

8. Pickuth D, Heywang-Kobrunner SH and Spielmann RP Computed tomography and magnetic resonance imaging features of olfactory neuroblastoma: an analysis of 22 cases. Clin Otolaryngol Allied Sci 24: 457-461, 1999.

9. Lund VJ,Howard D, Wei W and Spittle M: Olfactory neuroblastoma: Past, present, and future? Laryngoscope 113: 502-507, 2003.

10. Walch C, Stammberger H, Anderhuber W, Unger F, Kole W and Feichtinger K: The minimally invasive approach to olfactory neuroblastoma: combined endoscopic and stereotactic treatment. Laryngoscope 110: 635-640, 2000.

11. Kadish S, Goodman M and Wang CC: Olfactory neuroblastoma. A clinical analysis of 17 cases. Cancer 37: 1571-1576, 1976.

12. de Gabory L, Abdulkhaleq HM, Darrouzet V, Bébéar JP and Stoll D: Long-term results of 28 esthesioneuroblastomas managed over 35 years. Head Neck 33: 82-86, 2011.

13. Bhattacharyya N, Thornton AF, Joseph MP, Goodman ML and Amrein PC: Successful treatment of esthesioneuroblastoma and neuroendocrine carcinoma with combined chemotherapy and proton radiation. Results in 9 cases. Arch Otolaryngol Head Neck Surg 123: 34-40, 1997.
14. Howard DJ, Lund VJ and Wei WI: Craniofacial resection for tumors of the nasal cavity and paranasal sinuses: A 25-year experience. Head Neck 28: 867-873, 2006.

15. Morita A, Ebersold MJ, Olsen KD, Foote RL, Lewis JE and Quast LM: Esthesioneuroblastoma: prognosis and management. Neurosurgery 32: 706-714, 1993.

16. Aboziada MA and Eisbruch A: Esthesioneuroblastoma: the role of postoperative irradiation after complete surgical resection. J Egypt Natl Canc Inst 22: 143-148, 2010.

17. Benfari G, Fusconi M, Ciofalo A, Gallo A, Altissimi G, Celani T and De Vincentiis M: Radiotherapy alone for local tumour control in esthesioneuroblastoma. Acta Otorhinolaryngol Ital 28: 292-297, 2008

18. Demiroz C, Gutfeld O, Aboziada M, Brown D, Marentette LJ and Eisbruch A: Esthesioneuroblastoma: is there a need for elective neck treatment? Int J Radiat Oncol Biol Phys 15: 255-261, 2011.

19. Montava M, Verillaud B, Kania R, Sauvaget E, Bresson D, Mancini J, Froelich S and Herman P: Critical analysis of recurrences of esthesioneuroblastomas: can we prevent them? Eur Arch Otorhinolaryngol 271: 3215-3222, 2014. 